The study provides valuable information on a number rare disorders in the UAE. Early diagnosis and treatment leading to better prognosis is an important gain. Moreover, these results equip healthcare leaders with new information on the burden of these conditions to ensure better planning and provision of health services.

\section{CARER AND PATIENT PERSPECTIVE ON CLINICAL AND RESEARCH NEEDS TO IMPROVE CARE FOR FOOD PROTEIN INDUCED ENTEROCOLITIS}

Shubhasree Mukherjee, Rebecca Knibb, Ekaterina Khaleva, Marta Vazquez-Ortiz. UK

\subsection{6/bmjpo-2021-RCPCH.221}

Background Food Protein induced enterocolitis (FPIES) is a non-Immunoglobulin E(Ig-E) mediated gastrointestinal food allergy affecting mainly children. It has a significant impact on quality of life and despite being a potentially life-threatening allergy, awareness is poor and the diagnosis is often missed or delayed, as 'it does not look like an allergy' and IgE-based allergy tests are usually negative. There is very limited data on parent/patient perspectives of the unmet clinical or research needs in FPIES that can affect clinical outcomes.

Objectives To identify unmet research and clinical needs, as perceived by patients with FPIES and carers of children with FPIES, to deliver value-based patient care.

Methods An online survey was disseminated in English through the USA-based patient/parent association 'FPIES Foundation', FPIES UK, Food Allergy and Anaphylaxis Australia, Food Allergy Canada and Allergy Italia, between May 2020 and July 2020. Participants included adults with self-reported FPIES or a carer of a child (0-18 years) with FPIES. The survey collected demographic data of patients, incidence of acute and chronic FPIES, parent and patient perspectives of unmet research needs and psychological effect of FPIES on families, including ranking a number of potential needs from 1 (not essential) to 9 (extremely essential). The survey contained closed and open-ended questions; the latter were analysed using thematic analysis.

Results A total of 285 respondents completed the survey, of which $255(89.5 \%)$ were parent/carer of a child with FPIES (0-18 years), 21 (7.4\%) were adults with FPIES and 9 (3.2\%) were adult with FPIES themselves and a parent/care-giver of a child with FPIES. Female participants comprised 93\% (265/ $285)$ of the total and $49.5 \%(141 / 285)$ belonging to the age group of $35-44$ years.

Majority $(76 \%, 182 / 239)$ of children had FPIES to multiple foods, the commonest culprit food being grains other than gluten i.e. rice/oats $(62 \%, 148 / 239)$. 48.75\% (117/240) of children suffered from chronic with acute FPIES followed closely by children with acute FPIES only (46.25\%, 111/240).

The top research priorities identified by participants (in decreasing order of importance) were 1.) Creating awareness about FPIES amongst health care professionals(HCP), in particular emergency doctors and nurses; 2.) Education of medical students on FPIES, 3.) The development of a prognostic and predictive test for FPIES, 4) An established care plan for continuity of care following visit to emergency and 5) A diagnostic test to diagnose FPIES acutely against common differentials.

The major themes identified in the data were 1.) Creating awareness about FPIES amongst HCP and in community, 2.)
FPIES is psychologically stressful 3.) FPIES leads to social restriction due to fear of accidental ingestion leading to severe symptoms 4) FPIES is financially stressful and should be covered by health insurance and 5) Lack of stringent foodlabelling.

Conclusions Increasing awareness amongst healthcare professionals to avoid misdiagnosis/delay and lack of a prognostic/diagnostic test for FPIES were the key research needs. Further research and clinical improvement strategies should be focused on these areas.

\section{CHILDHOOD OBESITY: A SURVEY OF KNOWLEDGE AND PRACTICES OF PEDIATRICIANS AND PEDIATRIC RESIDENTS}

Li Ming Ong, Kai Guo Benny Loo, Jean Yin Oh, Elaine Chew. Singapore

\subsection{6/bmjpo-2021-RCPCH.222}

Background Childhood obesity is one of the most common and serious public health issues in the twenty-first century. Overweight and obese children are more likely to stay obese into adulthood and develop cardiovascular diseases at a younger age. Now, pediatricians have to manage these conditions that were once regarded as adult diseases.

Objectives The main aims are: 1. Identify knowledge gaps in medical, dietary, exercise and psychological aspects of childhood obesity; 2. Highlight the challenges in treating obese patients.

Methods This is a single-center, cross sectional quantitative survey of pediatricians and pediatric residents working in the largest tertiary children hospital in Singapore (KK Women's and Children's Hospital). Baseline demographic data as well as participants' knowledge in medical, dietary, physical activity and psychological aspects of childhood obesity were collected using a 32-question survey. A group of medical and allied health professionals designed and reviewed the survey. The correct answers for BMI cut-offs for obesity and overweight, healthy balanced diet in proportion of plate, recommended sugar intake, physical activity for children were defined according to national and international guidelines. Junior doctors comprised of house officers, medical officers and junior residents. Senior doctors were made up of senior residents and pediatricians.

Results A total of 123 doctors were surveyed (46\% were juniors). Both juniors and seniors tend to encounter overweight and obese patients on weekly basis. The most common challenge cited during patient consultations was patient motivation-related. Expectedly, the seniors perceived themselves to be more comfortable and knowledgeable compared to juniors. Other common challenges were lack of time and other medical conditions taking precedence over weight concerns. Physical activity recommendation was the weakest area, followed by dietary advice. There was no significant differences in medical, dietary, physical activity and psychological knowledge associated with obesity between junior and senior doctors.

Conclusions There were similar knowledge gaps in all domains of childhood obesity between junior and senior doctors. Physical activity recommendation is the weakest area. Patient motivation is the commonest challenge faced. Hence, future education programs should target all doctors and focus on motivational interviewing. 